Full length article

\title{
Bayesian methodology for target tracking using combined RSS and AoA measurements
}

\author{
Slavisa Tomic a,b,*, Marko Beko ${ }^{\text {b,c }}$, Rui Dinis ${ }^{\text {d,e }}$, Milan Tuba ${ }^{f}$, Nebojsa Bacanin ${ }^{f}$ \\ a ISR/IST, LARSyS, Universidade de Lisboa, 1049-001 Lisbon, Portugal \\ b CICANT-CIC.DIGITAL, Universidade Lusófona de Humanidades e Tecnologias, Campo Grande 376, 1749-024 Lisboa, Portugal \\ c CTS/UNINOVA, Campus da FCT/UNL, Monte de Caparica, 2829-516 Caparica, Portugal \\ ${ }^{\mathrm{d}}$ Instituto de Telecomunicações, Av. Rovisco Pais 1, Torre Norte, piso 10, 1049-001 Lisboa, Portugal \\ ${ }^{\text {e }}$ Dep. ${ }^{\circ}$ de Eng. ${ }^{a}$ Electrotécnica, FCT/UNL, 2829-516 Caparica, Portugal \\ ${ }^{\mathrm{f}}$ Faculty of Computer Science, John Naisbitt University, Belgrade, Serbia
}

\section{A R T I C L E I N F O}

\section{Article history:}

Received 12 February 2017

Received in revised form 23 July 2017

Accepted 11 October 2017

\section{Keywords:}

Target tracking

Received signal strength (RSS)

Angle of arrival (AoA)

Maximum a posteriori (MAP) estimator

Kalman filter (KF)

Extended KF (EKF)

Unscented KF (UKF)

Particle filter (PF)

\begin{abstract}
A B S T R A C T
This work addresses the target tracking problem based on received signal strength (RSS) and angle of arrival (AoA) measurements. The Bayesian methodology, which integrates the information given by observations with prior knowledge extracted from target motion model in order to enhance the estimation accuracy was employed. First, by converting the considered highly non-linear measurement model into a linear one, i.e., a novel linearization technique of the measurement model is proposed. The derived model is then merged with the prior knowledge, and a novel maximum a posteriori (MAP) estimator whose solution is given in closed-form is proposed. It is also shown that the Kalman filter (KF) can be directly applied on top of the linearized observation model, which results in a proposal of a novel KF algorithm. Furthermore, to the best of authors' knowledge, this paper premierly presents the application of the extended KF (EKF) and the unscented KF (UKF) to the considered tracking problem, by applying first-order linearization technique to the original non-linear model, and by applying the unscented transformation to carefully selected sample points, respectively. Finally, importance weights are computed for a large number of randomly selected sample points to render a well-known particle filter (PF) solution. Simulation results show that the proposed algorithms perform better than a naive one which uses only information from observations. They also confirm the effectiveness of the proposed linearization technique in comparison with the existing one, reducing the estimation error for about $25 \%$.
\end{abstract}

(c) 2017 Published by Elsevier B.V.

\section{Introduction}

Accurate localization of a moving target has become an important task because of its relevance in both military and commercial applications [1-5]. Cost, complexity and applicability in all terrain, limit the use of the existing location aware systems (e.g., GPS). Hence, new techniques to enhance accuracy and reduce the cost have been proposed by the researchers. Amongst other, these include localization algorithms based on received signal strength (RSS), time of arrival, angle of arrival (AoA) or a combination of them [5-15].

The work described in [7-15] considered the classical target localization problem, based on observations exclusively. In [2,3]

\footnotetext{
* Corresponding author at: ISR/IST, LARSyS, Universidade de Lisboa, 1049-001 Lisbon, Portugal.

E-mail addresses: stomic@isr.ist.utl.pt (S. Tomic),beko.marko@ulusofona.pt, mbeko@uninova.pt (M. Beko),rdinis@fct.unl.pt (R. Dinis), tuba@ieee.org (M. Tuba), nbacanin@megatrend.edu.rs (N. Bacanin).
}

and [5], the authors considered target tracking problem, where the observations were combined with some prior knowledge to enhance the estimation accuracy. However, they considered RSSbased target tracking problem only. The inventors of [6] tackled the target tracking problem by making use of radiated signal strength and the relatively measured radio carrier phase-delay to detect and position the radio transmitting device(s). In [4], the authors investigated the target tracking problem by employing hybrid, RSS and AoA, measurements. The authors first linearized the highly non-linear measurement model and on top of the linearized model they applied a Kalman filter (KF). In [4], the measurement model was linearized by using a very simple and intuitive approach. It can be summarized as forming a line and using the RSS measurements to determine the length of the line. At one point of the line, the authors situated a known anchor location and used the AoA to determine the slope of the line. In that way, an estimate of the target location was obtained at the other point of the line. Although this is an effective way to tackle the non-linearity of the measurement model, the authors in [4] treated all links as equal, 
and no mitigation technique was used to deal with potentially negative impact from distant links. Besides the $\mathrm{KF}$, a particle filter (PF) algorithm was also proposed in [4], as well as a generalized pattern search method for estimating the path loss exponent (PLE) for each link in every time step.

In this work, the target tracking problem by taking advantage of coupled RSS and AoA measurements is addressed. Various algorithms that are based on the Bayesian approach, which integrates the information gathered through radio observations with prior knowledge given by target state transition model are proposed. Therefore, the main contributions of this work are as follows. First, a novel linearization technique to efficiently represent the original non-linear measurement model with a linear one is proposed. The proposed linearization technique is fundamentally different from the existing one described in [4], since a Cartesian to polar (spherical for 3-dimensional space) coordinates conversion to deal effortlessly with the non-linear terms in the measurement model is used here. Moreover, in contrast to [4] where the authors treat all links as equal, here, weights which give more importance to nearby links and mitigate the potential negative impact of the remote ones are applied. This linearized measurement model is then combined with the prior knowledge to design a novel maximum a posteriori (MAP) estimator. Owing to the use of the derived measurement model, it is also shown that the application of the KF is straightforward, and thus, a novel KF algorithm is proposed. Although various extensions of the KF are available in the literature nowadays, to the best of authors' knowledge, none of them were applied to the considered RSS-AoA-based target tracking problem. Therefore, by applying first-order Taylor series expansion, the extended KF (EKF) is developed also. Furthermore, to capture the mean and covariance up to a higher order of Taylor series expansion, a number of carefully chosen sample points were used with the unscented transformation to build an unscented KF (UKF). Finally, a large number of sample points were chosen at random and importance weights were assigned to them by calculating the likelihood that these points correspond to a given observation in order to derive a PF.

This paper is organized as follows. In Section 2, we introduce the target state transition model as well as the measurement model, and we formulate the target tracking problem by using the Bayesian approach. Section 3 describes the proposed technique used to linearize the measurement model. Section 4 present the derivation of the proposed tracking algorithms. In Section 5, simulation results are presented for two different target trajectories in order to validate the performance of the proposed algorithms. Finally, Section 6 summarizes the main conclusions.

\section{Problem formulation}

Let $\boldsymbol{x}_{t}=\left[x_{\mathrm{x}}, x_{\mathrm{y}}\right]^{T}$ and $\boldsymbol{a}_{i}=\left[a_{i \mathrm{x}}, a_{i \mathrm{y}}\right]^{T}$, for $i=1, \ldots, N$ denote the unknown location of a moving target at time $t$ and known location of the $i$ th static anchor, respectively ${ }^{1}$. For simplicity, a constant velocity motion model is assumed here, perturbed only by slight speed corrections, so that the velocity components in the $\mathrm{x}$ and $\mathrm{y}$ directions at time $t$ are given by

$\boldsymbol{v}_{t}=\boldsymbol{v}_{t-1}+\boldsymbol{r}_{v_{t}}$,

where $\boldsymbol{r}_{v_{t}}$ represents the noise perturbations. Hence, from the equations of motion [16], the target location at time $t$ is

$\boldsymbol{x}_{t}=\boldsymbol{x}_{t-1}+\boldsymbol{v}_{t-1} \Delta+\boldsymbol{r}_{x_{t}}$,

where $\Delta$ and $\boldsymbol{r}_{x_{t}}$ are the sampling interval between two consecutive time steps and location process noise, respectively. Now, if

\footnotetext{
1 Without loss of generality, this paper focuses on 2-dimensional scenario. The extension to 3-dimensional scenario is straightforward.
}

the target state at $t$ is described by its location and velocity, i.e., $\boldsymbol{\theta}_{t}=\left[\boldsymbol{x}_{t}^{T}, \boldsymbol{v}_{t}^{T}\right]^{T}$, from (1) and (2) we get

$\boldsymbol{\theta}_{t}=\boldsymbol{S} \boldsymbol{\theta}_{t-1}+\boldsymbol{r}_{t}$

where $\boldsymbol{r}_{t}=\left[\boldsymbol{r}_{x_{t}}^{T}, \boldsymbol{r}_{v_{t}}^{T}\right]^{T}$ is the state process noise [1-5]. This process noise is assumed to be zero-mean Gaussian with a covariance matrix $\boldsymbol{Q}$, i.e., $\boldsymbol{r}_{t} \sim \mathcal{N}(\mathbf{0}, \boldsymbol{Q})$, where $\boldsymbol{Q}$ is defined as

$\boldsymbol{Q}=q\left[\begin{array}{cccc}\frac{\Delta^{3}}{3} & 0 & \frac{\Delta^{2}}{2} & 0 \\ 0 & \frac{\Delta^{3}}{3} & 0 & \frac{\Delta^{2}}{2} \\ \frac{\Delta^{2}}{2} & 0 & \Delta & 0 \\ 0 & \frac{\Delta^{2}}{2} & 0 & \Delta\end{array}\right]$,

with $q$ denoting the state process noise intensity $[1,3,17]$. The symbol $\boldsymbol{S}$ in (3) stands for the state transition matrix, given by

$\boldsymbol{S}=\left[\begin{array}{llll}1 & 0 & \Delta & 0 \\ 0 & 1 & 0 & \Delta \\ 0 & 0 & 1 & 0 \\ 0 & 0 & 0 & 1\end{array}\right]$.

At each time step, the target broadcasts a signal to anchors which then extract the RSS and AoA information from it. Hence, the measurement equation can be expressed as

$\boldsymbol{z}_{t}=\boldsymbol{h}\left(\boldsymbol{x}_{t}\right)+\boldsymbol{n}_{t}$,

where $\boldsymbol{z}_{t}=\left[\boldsymbol{P}_{t}^{T}, \boldsymbol{\phi}_{t}^{T}\right]^{T}\left(\boldsymbol{z}_{t} \in \mathbb{R}^{2 N}\right)$ is the observation vector composed of RSS, $\boldsymbol{P}_{t}=\left[P_{i}^{(t)}\right]^{T}$, and AoA, $\boldsymbol{\phi}_{t}=\left[\phi_{i}^{(t)}\right]^{T}$, measurements at time $t$. The function $\boldsymbol{h}\left(\boldsymbol{x}_{t}\right)$ in (4) is defined as $h_{i}\left(\boldsymbol{x}_{t}\right)=P_{0}-$ $10 \gamma \log _{10} \frac{d_{i}}{d_{0}}$ for $i=1, \ldots, N[18]$, where $P_{0}(\mathrm{dBm})$ is the reference power at a distance $d_{0}\left(d_{0} \leq d_{i}\right), \gamma$ is the path loss exponent (PLE) and $d_{i}$ is the distance between the target and the $i$ th anchor, and $h_{i}\left(\boldsymbol{x}_{t}\right)=\tan ^{-1}\left(\frac{x_{\mathrm{y}}-a_{i \mathrm{y}}}{x_{\mathrm{x}}-a_{i \mathrm{x}}}\right)$ for $i=N+1, \ldots, 2 N$ [19]. The measurement noise is modeled as $\boldsymbol{n}_{t} \sim \mathcal{N}(\mathbf{0}, \boldsymbol{C})$, where the noise covariance is defined as $\boldsymbol{C}=\operatorname{diag}\left(\left[\sigma_{n_{i}}^{2}, \sigma_{m_{i}}^{2}\right]\right) \otimes \boldsymbol{I}_{4}$, with $\sigma_{n_{i}}^{2}(\mathrm{~dB})$ and $\sigma_{m_{i}}^{2}$ (rad) being the variances of the RSS and AoA measurement noise, respectively, $\boldsymbol{I}_{M}$ representing the identity matrix of size $M$ and symbol $\otimes$ denoting the Kronecker product.

Our goal is to incorporate the prior knowledge, given by the state transition model (3) to construct the estimation of the state at the next time instant, into our estimator. This approach is known as the Bayesian approach [16], and it can improve the estimation accuracy in comparison with the classical approach which disregards the prior knowledge. By using (3) and (4), we can build the marginal posterior probability distribution function (PDF), $p\left(\boldsymbol{\theta}_{t} \mid \boldsymbol{z}_{1: t}\right)$, to quantify the belief we have in the values of the state $\boldsymbol{\theta}_{t}$ given all the past measurements $\boldsymbol{z}_{1: t}$. From $p\left(\boldsymbol{\theta}_{t} \mid \boldsymbol{z}_{1: t}\right)$, we can obtain an estimate at any time step we desire.

Below, a recursive approach for the evaluation of $p\left(\boldsymbol{\theta}_{t} \mid \boldsymbol{z}_{1: t}\right)$ at any time instant is presented, which is typical for Bayesian methods [1-5].

- Initialization: The marginal posterior PDF at $t=0$ is set to the prior PDF $p\left(\boldsymbol{\theta}_{0}\right)$ of $\boldsymbol{\theta}_{0}$.

- Prediction: By using the state transition model (3), the predictive PDF of the state at $t$ is given by

$p\left(\boldsymbol{\theta}_{t} \mid \boldsymbol{z}_{1: t-1}\right)=\int p\left(\boldsymbol{\theta}_{t} \mid \boldsymbol{\theta}_{t-1}\right) p\left(\boldsymbol{\theta}_{t-1} \mid \boldsymbol{z}_{1: t-1}\right) d \boldsymbol{\theta}_{t-1}$.

- Update: By following the Bayes' rule $[1,17]$, we have

$$
p\left(\boldsymbol{\theta}_{t} \mid \boldsymbol{z}_{1: t}\right)=\frac{p\left(\boldsymbol{z}_{t} \mid \boldsymbol{\theta}_{t}\right) p\left(\boldsymbol{\theta}_{t} \mid \boldsymbol{z}_{1: t-1}\right)}{\int p\left(\boldsymbol{z}_{t} \mid \boldsymbol{\theta}_{t}\right) p\left(\boldsymbol{\theta}_{t} \mid \boldsymbol{z}_{1: t-1}\right) d \boldsymbol{\theta}_{t}} .
$$


Note that the denominator in (6) is just a normalizing constant. In general, the marginal PDF at $t-1$ cannot be calculated analytically, and the integral in (5) cannot be obtained analytically if the state model is non-linear. Therefore, some approximations are required in order to obtain $p\left(\boldsymbol{\theta}_{t} \mid \boldsymbol{z}_{1: t}\right)$.

\section{Linearization of the observation model}

Assuming that the noise term is sufficiently small, from (4) it can be written for the RSS measurement model

$\lambda_{i}\left\|\boldsymbol{x}_{t}-\boldsymbol{a}_{i}\right\| \approx \eta d_{0}$, for $i=1, \ldots, N$,

where $\lambda_{i}=10^{\frac{P_{i}^{(t)}}{10 \gamma}}, \eta=10^{\frac{P_{0}}{10 \gamma}}$. According to Cartesian to polar coordinates conversion, one can express $\boldsymbol{x}_{t}-\boldsymbol{a}_{i}=r_{i} \boldsymbol{u}_{i}: r_{i} \geq$ $0,\left\|\boldsymbol{u}_{i}\right\|=1$, where the unit vector can be found by using the available AoA information, i.e., $\boldsymbol{u}_{i}=\left[\cos \left(\phi_{i}^{(t)}\right), \sin \left(\phi_{i}^{(t)}\right)\right]^{T}$. Applying this conversion in (7) and multiplying the left hand side of (7) by $\boldsymbol{u}_{i}^{T} \boldsymbol{u}_{i}$, yields

$\lambda_{i} \boldsymbol{u}_{i}^{T}\left(\boldsymbol{x}-\boldsymbol{a}_{i}\right) \approx \eta d_{0}$

From (4), similar can be done for the AoA measurement model

$\boldsymbol{c}_{i}^{T}\left(\boldsymbol{x}-\boldsymbol{a}_{i}\right) \approx 0$, for $i=N+1, \ldots, 2 N$,

where $\boldsymbol{c}_{i}=\left[-\sin \left(\phi_{i}^{(t)}\right), \cos \left(\phi_{i}^{(t)}\right)\right]^{T}$.

By introducing weights, $\boldsymbol{w}=\left[w_{i}\right]^{T}$, in (8) and (9) such that $w_{i}=$ $1-\frac{\widehat{d}_{i}}{\sum_{i=1}^{N} \widehat{d}_{i}}$ and $\widehat{d}_{i}=d_{0} 10^{\frac{P_{0}-P_{i}^{(t)}}{10 \gamma}}$, in order that more importance is given to nearby links, yields respectively

$w_{i} \lambda_{i} \boldsymbol{u}_{i}^{T}\left(\boldsymbol{x}_{t}-\boldsymbol{a}_{i}\right) \approx w_{i} \eta d_{0}$, for $i=1, \ldots, N$,

$w_{i} \boldsymbol{c}_{i}^{T}\left(\boldsymbol{x}_{t}-\boldsymbol{a}_{i}\right) \approx 0$, for $i=N+1, \ldots, 2 N$.

Thus, the RSS and AoA measurement models can be linearized by (10) and written in a vector form as a function of the target state as

$\boldsymbol{A} \boldsymbol{\theta}_{t}=\boldsymbol{b}$.

where

$\boldsymbol{A}=\left[\begin{array}{ccc}\vdots & \vdots & \vdots \\ w_{i} \lambda_{i} \boldsymbol{u}_{i}^{T} & 0 & 0 \\ \vdots & \vdots & \vdots \\ w_{i} \mathbf{c}_{i}^{T} & 0 & 0 \\ \vdots & \vdots & \vdots\end{array}\right], \boldsymbol{b}=\left[\begin{array}{c}\vdots \\ w_{i}\left(\lambda_{i} \boldsymbol{u}_{i}^{T} \boldsymbol{a}_{i}+\eta d_{0}\right) \\ \vdots \\ w_{i} \boldsymbol{c}_{i}^{T} \boldsymbol{a}_{i} \\ \vdots\end{array}\right]$,

By applying the least squares (LS) principle to (11), a target state estimate can be obtained as

$\hat{\boldsymbol{\theta}}_{t \mid t}=\underset{\boldsymbol{\theta}_{t}}{\arg \min }\left\|\boldsymbol{A} \boldsymbol{\theta}_{t}-\boldsymbol{b}\right\|^{2}$

whole solution is readily found as $\hat{\boldsymbol{\theta}}_{t \mid t}=\left(\boldsymbol{A}^{T} \boldsymbol{A}\right)^{-1}\left(\boldsymbol{A}^{T} \boldsymbol{b}\right)$.

In order to show that by employing the proposed linearization technique, one gets a tight approximation of the maximum likelihood (ML) estimator, we call the reader's attention to Fig. 1. In this figure, a possible realization of the ML and the LS in (12) objective functions are plotted, when all sensors were randomly deployed in a square region with a border of $B=20 \mathrm{~m}$. The true target location was set at $[5.09 ; 2.11]$ and $N=5$ anchors were deployed at $[6.36,18.80,9.59,10.89,10.88,10.45 ; 2.38$, $12.91,12.79,12.95,14.42,19.87]$, and the rest of the parameters are set as in Section 5. Fig. 1 shows that the optimal solutions of the two functions are close to each other, meaning that the

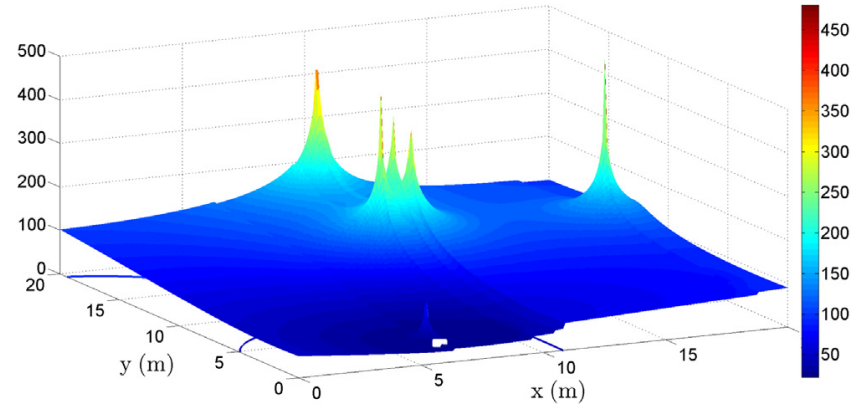

(a) ML objective function.

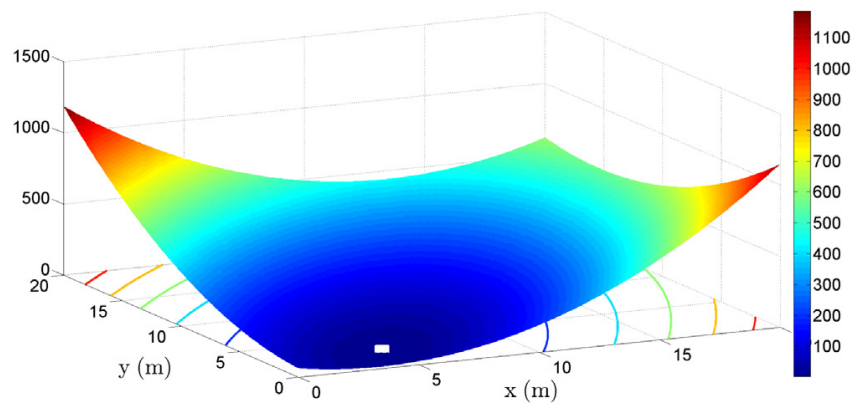

(b) WLS objective function in (12)

Fig. 1. Illustration of the objective functions versus $x(m)$ and $y(m)$ coordinates, when $N=5, \sigma_{n_{i}}=9 \mathrm{~dB}, \sigma_{m_{i}}=4$ degrees, $\gamma=3, \gamma_{i} \sim \mathcal{U}[2.7,3.3], P_{0}=-10 \mathrm{dBm}$ the minimum of the objective function is indicated by a white square.

LS objective function (i.e., the new linearization technique) is an excellent approximation of the ML one. Please note that it is so, even for the case when the assumption that the noise power is low does not hold. However, the objective function in (12) is much smoother than the ML one, which allows us to easily obtain its global minimum.

\section{The proposed methods}

\subsection{MAP-based estimator}

From $p\left(\boldsymbol{\theta}_{t} \mid \boldsymbol{z}_{1: t}\right)$, a state estimate, $\hat{\boldsymbol{\theta}}_{t \mid t}$, of $\boldsymbol{\theta}_{t}$ can be obtained according to the maximum a posteriori (MAP) criteria [16], by maximizing the marginal posterior PDF, i.e.,

$\hat{\boldsymbol{\theta}}_{t \mid t}=\underset{\boldsymbol{\theta}_{t}}{\arg \max } p\left(\boldsymbol{\theta}_{t} \mid \boldsymbol{z}_{1: t}\right) \approx \underset{\boldsymbol{\theta}_{t}}{\arg \max } p\left(\boldsymbol{z}_{t} \mid \boldsymbol{\theta}_{t}\right) p\left(\boldsymbol{\theta}_{t} \mid \boldsymbol{z}_{1: t-1}\right)$.

This is reminiscent of the ML estimator, except for the existence of the prior PDF. The problem in (13) is highly non-convex and its analytical solution cannot be obtained in general. Thus, (13) is approximated by another estimator whose solution is readily obtained.

First, assume that $p\left(\boldsymbol{\theta}_{t-1} \mid \boldsymbol{z}_{1: t-1}\right)$ has Gaussian distribution with mean $\hat{\boldsymbol{\theta}}_{t-1 \mid t-1}$ and covariance $\hat{\boldsymbol{P}}_{t-1 \mid t-1}$ (a similar assumption was made in [17]). According to (5), we obtain

$p\left(\boldsymbol{\theta}_{t} \mid \boldsymbol{z}_{1: t-1}\right) \approx \frac{1}{k_{1}} \exp \left\{-\frac{1}{2}\left(\boldsymbol{\theta}_{t}-\hat{\boldsymbol{\theta}}_{t \mid t-1}\right)^{T} \hat{\boldsymbol{P}}_{t \mid t-1}^{-1}\left(\boldsymbol{\theta}_{t}-\hat{\boldsymbol{\theta}}_{t \mid t-1}\right)\right\}$,

where $k_{1}$ is a constant, and $\hat{\boldsymbol{\theta}}_{t \mid t-1}$ and $\hat{\boldsymbol{P}}_{t \mid t-1}$ are the mean and the covariance of the one-step predicted state, respectively, obtained through (3) as

$$
\begin{gathered}
\hat{\boldsymbol{\theta}}_{t \mid t-1}=\boldsymbol{S} \hat{\boldsymbol{\theta}}_{t-1 \mid t-1}, \\
\hat{\boldsymbol{P}}_{t \mid t-1}=\boldsymbol{S} \hat{\boldsymbol{P}}_{t-1 \mid t-1} \boldsymbol{S}^{T}+\boldsymbol{Q} .
\end{gathered}
$$


The likelihood function can be written as

$p\left(\boldsymbol{z}_{t} \mid \boldsymbol{\theta}_{t}\right)=\frac{1}{k_{2}} \exp \left(-\frac{1}{2}\left(\boldsymbol{z}_{t}-\boldsymbol{h}\left(\boldsymbol{x}_{t}\right)\right)^{T} \boldsymbol{C}^{-1}\left(\boldsymbol{z}_{t}-\boldsymbol{h}\left(\boldsymbol{x}_{t}\right)\right)\right)$,

where $k_{2}$ is a constant. In Section 3, it was shown how to tightly approximate (16) by another, linear estimator. Therefore, (13) can be written as

$$
\begin{aligned}
\hat{\boldsymbol{\theta}}_{t \mid t} & =\underset{\boldsymbol{\theta}_{t}}{\arg \min }\left(\boldsymbol{z}_{t}-\boldsymbol{h}\left(\boldsymbol{x}_{t}\right)\right)^{T} \boldsymbol{C}^{-1}\left(\boldsymbol{z}_{t}-\boldsymbol{h}\left(\boldsymbol{x}_{t}\right)\right) \\
& +\left(\boldsymbol{\theta}_{t}-\hat{\boldsymbol{\theta}}_{t \mid t-1}\right)^{T} \hat{\boldsymbol{P}}_{t \mid t-1}^{-1}\left(\boldsymbol{\theta}_{t}-\hat{\boldsymbol{\theta}}_{t \mid t-1}\right) .
\end{aligned}
$$

By following a similar approach as in (12), the problem (17) can be approximated by

$\hat{\boldsymbol{\theta}}_{t \mid t}=\underset{\boldsymbol{\theta}_{t}}{\arg \min }\left\|\widetilde{\boldsymbol{A}} \boldsymbol{\theta}_{t}-\widetilde{\boldsymbol{b}}\right\|^{2}$,

whose solution is readily given by

$\hat{\boldsymbol{\theta}}_{t \mid t}=\left(\widetilde{\boldsymbol{A}}^{T} \widetilde{\boldsymbol{A}}\right)^{-1}\left(\widetilde{\boldsymbol{A}}^{T} \widetilde{\boldsymbol{b}}\right)$,

where $\tilde{\boldsymbol{A}}=\left[\boldsymbol{A} ; \hat{\boldsymbol{P}}_{t \mid t-1}^{-\frac{1}{2}}\right]$ and $\widetilde{\boldsymbol{b}}=\left[\boldsymbol{b} ; \hat{\boldsymbol{P}}_{t \mid t-1}^{-\frac{1}{2}} \hat{\boldsymbol{\theta}}_{t \mid t-1}\right]$.

The step by step proposed MAP-based algorithm² is outlined in Algorithm 1.

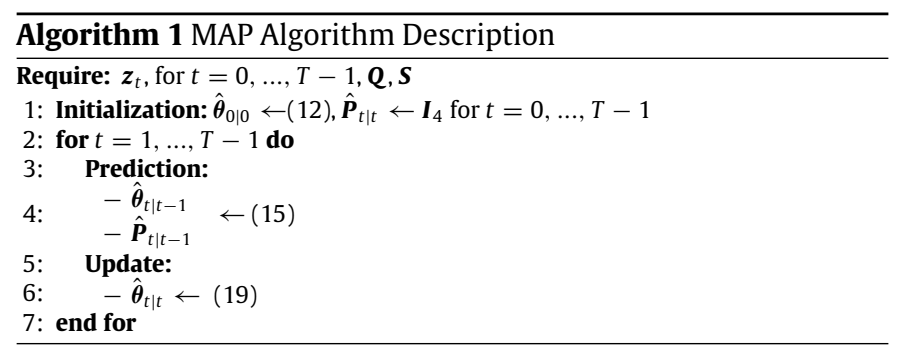

\subsection{Kalman filter}

When both the state and observation models are linear and the noise is assumed to be zero-mean with finite covariance, the KF provides the optimal solution in the least squares sense [1]. Although the observation model (4) is non-linear, it can be linearized according to (11). Then, the mean and the covariance are updated according to the $\mathrm{KF}[16]$, i.e.,

$$
\begin{gathered}
\hat{\boldsymbol{\theta}}_{t \mid t}=\hat{\boldsymbol{\theta}}_{t \mid t-1}+\boldsymbol{K}_{t}\left(\boldsymbol{b}-\boldsymbol{A} \hat{\boldsymbol{\theta}}_{t \mid t-1}\right), \\
\hat{\boldsymbol{P}}_{t \mid t}=\left(\boldsymbol{I}_{4}-\boldsymbol{K}_{t} \boldsymbol{A}\right) \hat{\boldsymbol{P}}_{t \mid t-1},
\end{gathered}
$$

where $\boldsymbol{K}_{t}$ is the Kalman gain at $t$.

The step by step proposed KF algorithm ${ }^{3}$ is outlined in Algorithm 2.

\subsection{Extended Kalman filter}

The EKF requires no assumptions about the linearity of the state or observation models. Instead, it approximates the non-linear

\footnotetext{
2 Note that an update of the state covariance matrix is not considered here. Even thought this could have been done fairly easily by taking advantage of the solution obtained by solving (19) and applying Karush-Kuhn-Tucker conditions to get an update on the state covariance (similar as in [17]), our simulations showed that this approach offered only marginal improvement for the MAP estimator; thus, this approach was not applied here.

3 It is worth noting that the KF estimator could also be derived directly from (17) by simply setting the derivative of (17) to zero and then calculating the updated covariance [16] as: $\hat{\boldsymbol{P}}_{t \mid t}=\mathbb{E}\left[\hat{\boldsymbol{\theta}}_{t \mid t} \hat{\boldsymbol{\theta}}_{t \mid t}^{T}\right]$, at each time step $t$. However, to do so, one would have to assume the measurement noise covariance perfectly known. Since this assumption might not hold in practice, and the measurement model is linearized, a different estimator whose closed-form solution is readily available (19) was derived instead.
}

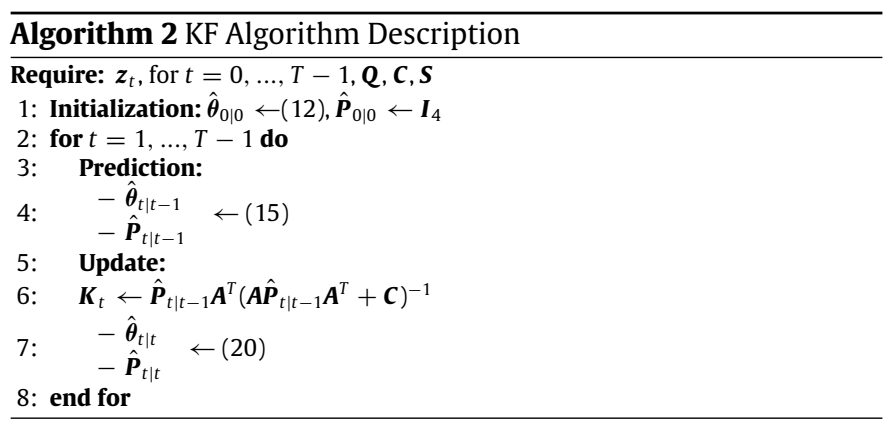

models by their first order Taylor series expansion ${ }^{4}$ [16]. Therefore, the state and state covariance update for the EKF is given by

$$
\begin{gathered}
\hat{\boldsymbol{\theta}}_{t \mid t}=\hat{\boldsymbol{\theta}}_{t \mid t-1}+\boldsymbol{K}_{t}\left(\boldsymbol{z}_{t}-\boldsymbol{h}\left(\boldsymbol{x} \mid \hat{\boldsymbol{x}}_{t \mid t-1}\right)\right), \\
\hat{\boldsymbol{P}}_{t \mid t}=\left(\boldsymbol{I}_{4}-\boldsymbol{K}_{t} \boldsymbol{J}_{\left.h(\boldsymbol{\theta}) \mid \hat{\boldsymbol{x}}_{t \mid t-1}\right)}\right) \hat{\boldsymbol{P}}_{t \mid t-1},
\end{gathered}
$$

where $\hat{\boldsymbol{x}}_{t \mid t-1}$ is the one-step predicted target location, $\boldsymbol{h}\left(\boldsymbol{x} \mid \hat{\boldsymbol{x}}_{t \mid t-1}\right)$ is

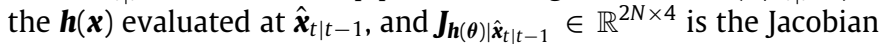
of $\boldsymbol{h}(\boldsymbol{\theta})$ evaluated at $\hat{\boldsymbol{x}}_{t \mid t-1}$, i.e.,

$\boldsymbol{J}_{\boldsymbol{h}(\theta) \mid \hat{\boldsymbol{x}}_{t \mid t-1}}=\left[\begin{array}{cccc}\vdots & \vdots & \vdots & \vdots \\ \xi \frac{\hat{x}_{\mathrm{x}, t \mid t-1}-a_{\mathrm{x} i}}{\left\|\hat{\boldsymbol{x}}_{t \mid t-1}-\boldsymbol{a}_{i}\right\|^{2}} & \xi \frac{\hat{x}_{\mathrm{y}, t \mid t-1}-a_{\mathrm{y} i}}{\left\|\hat{\boldsymbol{x}}_{t \mid t-1}-\boldsymbol{a}_{i}\right\|^{2}} & 0 & 0 \\ \vdots & \vdots & \vdots & \vdots \\ -\frac{\hat{x}_{\mathrm{y}, t \mid t-1}-a_{\mathrm{y} i}}{\left\|\hat{\boldsymbol{x}}_{t \mid t-1}-\boldsymbol{a}_{i}\right\|^{2}} & \frac{\hat{x}_{\mathrm{x}, t \mid t-1}-a_{\mathrm{x} i}}{\left\|\hat{\boldsymbol{x}}_{t \mid t-1}-\boldsymbol{a}_{i}\right\|^{2}} & 0 & 0 \\ \vdots & \vdots & \vdots & \vdots\end{array}\right]$,

with $\xi=\frac{10 d_{0} \gamma}{\ln (10)}$. For the sake of simplicity, we denote $\boldsymbol{J}_{\boldsymbol{h}(\boldsymbol{\theta}) \mid \hat{\boldsymbol{x}}_{t \mid t-1}}$ as $\boldsymbol{J}$.

The step by step proposed EKF algorithm is outlined in Algorithm 3.

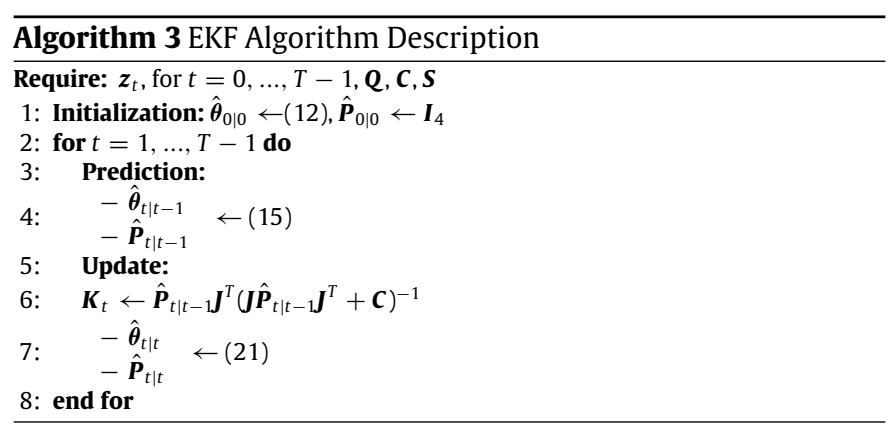

\subsection{Unscented Kalman filter}

The UKF utilizes the unscented transformation, which is founded on the intuition that it is easier to approximate a probability distribution than it is to approximate an arbitrary non-linear function or transformation [20]. The basic idea is to represent the state distribution by a random variable, which is described by a minimal set of carefully selected points, called sigma points. These points completely capture the mean and covariance of the random variable, and when passed through the non-linear system, they capture the mean and covariance up to the 3rd order (Taylor series expansion) for any non-linearity [20]. The sigma points, $\chi_{i}$, and

\footnotetext{
4 However, since a linear transition model (3) is considered here, no approximation of the state model is required.
} 
their corresponding weights, $w_{i}$, at $t$ are given by

$$
\begin{gathered}
\chi_{0}=\hat{\boldsymbol{\theta}}_{t-1 \mid t-1}, \\
\chi_{i}=\chi_{0}+\left(\sqrt{L+\kappa \hat{\boldsymbol{P}}_{t-1 \mid t-1}}\right), i=1, \ldots, L, \\
\chi_{i}=\chi_{0}-\left(\sqrt{L+\kappa \hat{\boldsymbol{P}}_{t-1 \mid t-1}}\right)_{i}, i=L+1, \ldots, 2 L, \\
w_{0}^{(m)}=\frac{\kappa}{\kappa+L}, \\
w_{0}^{(c)}=w_{0}^{(m)}+\left(1-\varrho^{2}+\beta\right), \\
w_{i}^{(m)}=w_{i}^{(c)}=\frac{1}{2(\kappa+L)}, i=1, \ldots, 2 L,
\end{gathered}
$$

where $\kappa=\varrho^{2}(L+\zeta)-L$ is a scaling parameter, $L$ is the size of $\hat{\boldsymbol{\theta}}_{t-1 \mid t-1}, \varrho=10^{-3}$ determines the spread of sigma points around the mean, $\zeta=0$ is a secondary scaling parameter, $\beta=2$ is used to incorporate prior knowledge of the distribution of $\boldsymbol{\theta}$, and $\left(\sqrt{L+\kappa \hat{\boldsymbol{P}}_{t-1 \mid t-1}}\right)_{i}$ is the $i$ th column of the matrix square root. These sigma points are then propagated through the system model, i.e.,

$\hat{\chi}_{i}=S \chi_{i}, \quad i=0, \ldots, 2 L$.

The predicted mean and covariance are obtained as [20-24]

$$
\begin{gathered}
\hat{\boldsymbol{\theta}}_{t \mid t-1}=\sum_{i=0}^{2 L} w_{i}^{(m)} \hat{\chi}_{i}, \\
\hat{\boldsymbol{P}}_{t \mid t-1}=\sum_{i=0}^{2 L} w_{i}^{(c)}\left(\hat{\boldsymbol{\chi}}_{i}-\hat{\boldsymbol{\theta}}_{t \mid t-1}\right)\left(\hat{\boldsymbol{\chi}}_{i}-\hat{\boldsymbol{\theta}}_{t \mid t-1}\right)^{T}+\boldsymbol{Q} .
\end{gathered}
$$

Similar is done with the measurement model. First, the predicted sigma points are passed through the observation model, and the predicted observation, innovation covariance and cross covariance are respectively computed as

$$
\begin{gathered}
\hat{\boldsymbol{z}}_{t \mid t-1}=\sum_{i=0}^{2 L} w_{i}^{(m)} h\left(\boldsymbol{x} \mid \hat{\chi}_{i}\right), \\
\hat{\boldsymbol{P}}_{t}^{Z Z}=\sum_{i=0}^{2 L} w_{i}^{(c)}\left(h\left(\boldsymbol{x} \mid \hat{\chi}_{i}\right)-\hat{\boldsymbol{z}}_{t \mid t-1}\right)\left(h\left(\boldsymbol{x} \mid \hat{\chi}_{i}\right)-\hat{\boldsymbol{z}}_{t \mid t-1}\right)^{T}+\boldsymbol{C}, \\
\hat{\boldsymbol{P}}_{t}^{\Theta Z}=\sum_{i=0}^{2 L} w_{i}^{(c)}\left(\hat{\boldsymbol{\chi}}_{i}-\hat{\boldsymbol{\theta}}_{t \mid t-1}\right)\left(h\left(\boldsymbol{x} \mid \hat{\chi}_{i}\right)-\hat{\boldsymbol{z}}_{t \mid t-1}\right)^{T} .
\end{gathered}
$$

Finally, the state and covariance update is obtained as

$$
\begin{gathered}
\hat{\boldsymbol{\theta}}_{t \mid t}=\hat{\boldsymbol{\theta}}_{t \mid t-1}+\boldsymbol{K}_{t}\left(\boldsymbol{z}_{t}-\hat{\boldsymbol{z}}_{t \mid t-1}\right), \\
\hat{\boldsymbol{P}}_{t \mid t}=\hat{\boldsymbol{P}}_{t \mid t-1}-\boldsymbol{K}_{t} \hat{\boldsymbol{P}}_{t}^{Z Z} \boldsymbol{K}_{t}^{T} .
\end{gathered}
$$

The step by step proposed UKF algorithm is outlined in Algorithm 4.

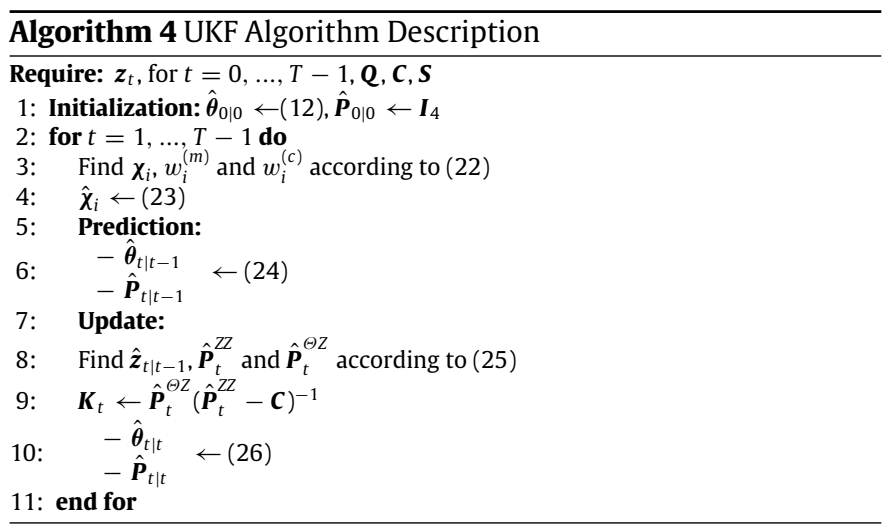

\subsection{Particle filter}

Similar as the UKF, the PF approximates the posterior PDF of the state with sample points, called particles, but with essential difference that these particles are selected at random. Essentially, it is nothing else but an ordinary randomization technique whose performance and computational complexity are directly proportional to the number of particles used $[2,4]$. The particles are iteratively updated according to new observations, and no linearity nor Gaussianity assumptions are required [24,25].

First, at $t, N_{p}$ particles are randomly generated, $\boldsymbol{p}_{t}^{n}$, according to the transition prior

$\hat{\boldsymbol{p}}_{t \mid t-1}^{n} \sim \mathcal{N}\left(\boldsymbol{S} \hat{\boldsymbol{p}}_{t-1 \mid t-1}^{n}, \boldsymbol{Q}\right), n=1, \ldots, N_{p}$.

Then, the likelihood that each particle corresponds to a given observations is evaluated, i.e.,

$\omega_{t}^{n}=\omega_{t-1}^{n} p\left(\boldsymbol{z}_{t} \mid \hat{\boldsymbol{p}}_{t \mid t-1}^{n}\right)$.

In this process, some particles might be given very low importance weights, $\omega_{t}^{n}$, for them to be negligible. Hence, these particles are discarded, and the samples with big weights are duplicated. This technique is known as resampling method [24,25], and is very common for PF.

After resampling, the weights are normalized, so that they sum up to 1 . The state update is calculated as the mean of the posterior, by using the normalized weights, $\tilde{\omega}_{t}^{n}$, and resampled particles, $\hat{\boldsymbol{p}}_{t \mid t}$, i.e.,

$\hat{\boldsymbol{\theta}}_{t \mid t}=\frac{1}{N_{p}} \sum_{n=1}^{N_{p}} \tilde{\omega}_{t}^{n} \hat{\boldsymbol{p}}_{t \mid t}^{n}$ 5.

The step by step proposed PF algorithm is outlined in Algorithm

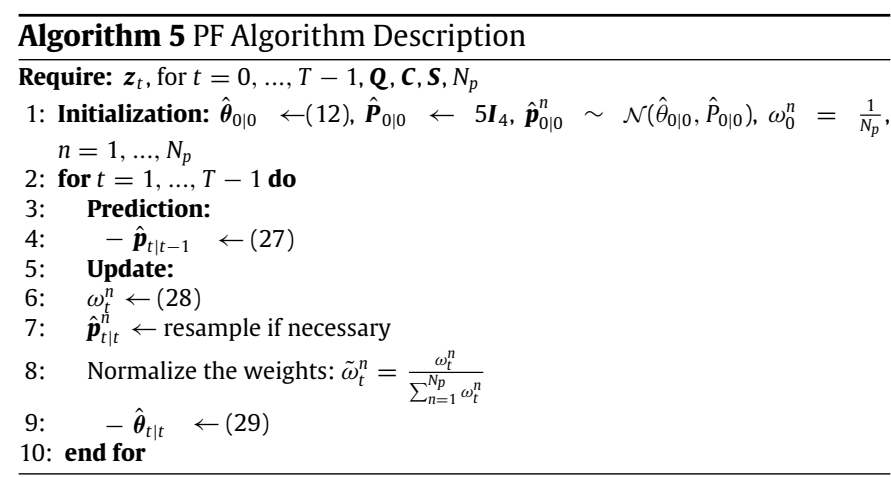

\section{Performance results}

The validity of the proposed algorithms is evaluated through computer simulations. Two essentially different scenarios are considered, in which the target takes sharp maneuvers and a more smooth one. The target state transition follows the state model (3), and at each time instant the radio measurements are generated according to (4). In both scenarios, the reference distance is set to $d_{0}=1 \mathrm{~m}$, the reference power to $P_{0}=-10 \mathrm{dBm}$, the path loss exponent to $\gamma=3$, and $N=3$ anchors are fixed at $\left[[70,10]^{T},[40,70]^{T}[10,40]^{T}\right]$. A sample is taken every $\Delta=1$ s during $T=150 \mathrm{~s}$ trajectory duration in each Monte Carlo, $M_{c}=1000$, run. Furthermore, $\sigma_{n_{i}}=9 \mathrm{~dB}, \sigma_{m_{i}}=4$ degrees, and $q=2.5 \times 10^{-3} \mathrm{~m}^{2} / \mathrm{s}^{3}$. For the proposed PF algorithm, $N_{p}=200$ is used, and the tuning factor is set to $q=2.5 \times 10^{-1} \mathrm{~m}^{2} / \mathrm{s}^{3}$ in order to allow greater search space to the particles. The performance metric used here is the root mean square error (RMSE), defined as 


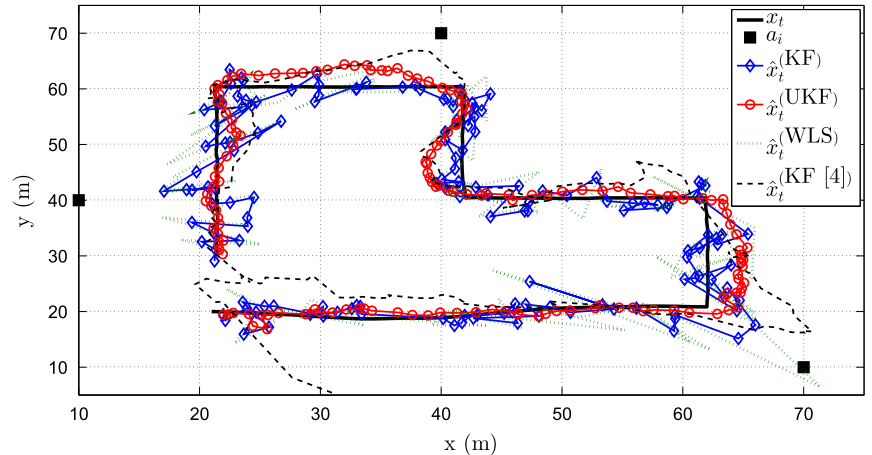

(a) Via KF, UKF, WLS, and KF [4].

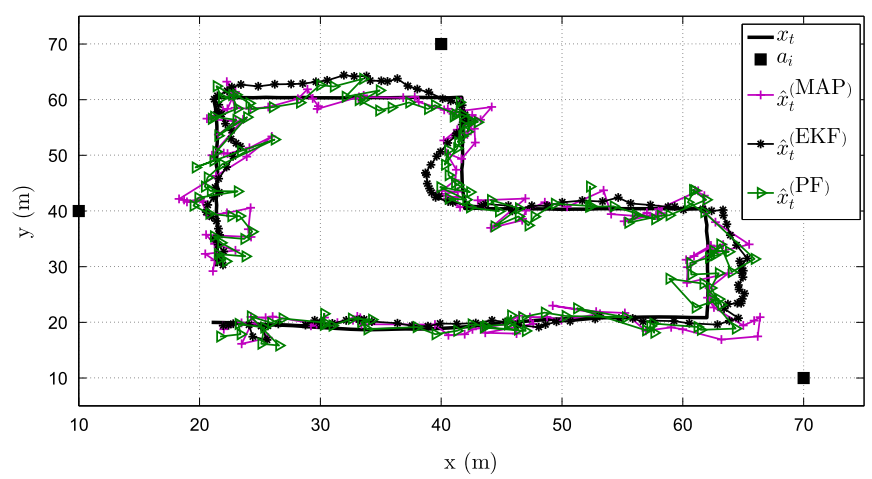

(b) Via MAP, EKF and PF.

Fig. 2. The true target trajectory in scenario 1, compared with the estimated trajectory.

$\operatorname{RMSE}_{t}=\sqrt{\sum_{i=1}^{M_{c}} \frac{\left\|\boldsymbol{x}_{i, t}-\widehat{\boldsymbol{x}}_{i, t}\right\|^{2}}{M_{c}}}$, where $\widehat{\boldsymbol{x}}_{i, t}$ denotes the estimate of the true target location, $\boldsymbol{x}_{i, t}$, in the $i$ th $M_{c}$ run at time instant $t$.

The proposed algorithms described in Section 4 are compared with the KF [4], where the initial target state was estimated by solving the LS method used in [4] to linearize the observation modep $\bar{F}$ , and the sequential WLS localization method [15] applied here to linearize the observation model, which does not take advantage of the prior knowledge.

The first scenario is illustrated in Fig. 2, where the initial target location is at $[21,20]^{T}$. From the figure, one can observe that all algorithms, except the WLS one, perform fairly good in the first phase $(t \leq 40)$ of the target trajectory. The reason for this behavior is that the trajectory is relatively smooth at this phase, and all algorithms benefit from the prior knowledge, except the WLS which is based purely on the quality of the observations. Furthermore, Fig. 2 exhibits a slight performance deterioration of all algorithms after each sharp maneuver of the target, especially as the target approaches any of the anchors. This is somewhat expected since the sudden turn of the target diminishes the prior knowledge stipulated by all tracking algorithms, and the proximity of an anchor intensifies the importance of that particular observation in comparison with the other ones. However, it can be seen that these impairments are mild, and that all tracking algorithms quickly recover from them.

The second scenario is illustrated in Fig. 3, where the initial target location is at $[35,15]^{T}$. It can be seen that, when the target constantly changes its direction, but without particularly sharp maneuvers, the performance of the tracking algorithm betters in

\footnotetext{
5 In order to make the comparison fair, the true value of the PLE is given to the $\mathrm{KF}[4]$ at every time step.
}

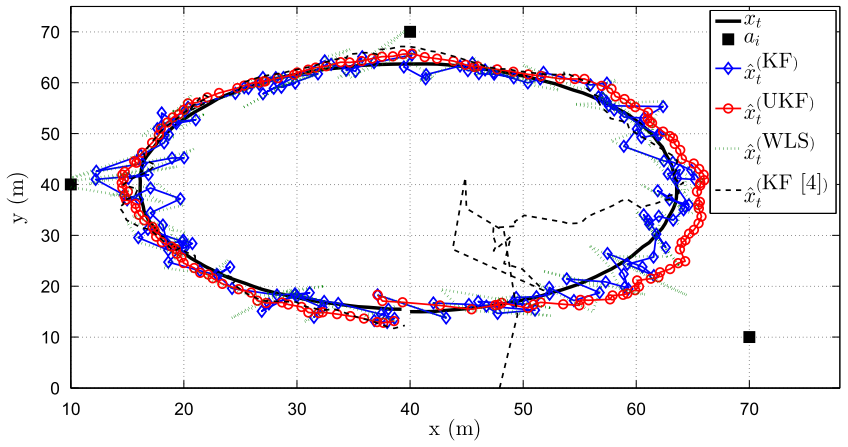

(a) Via KF, UKF, WLS, and KF [4].

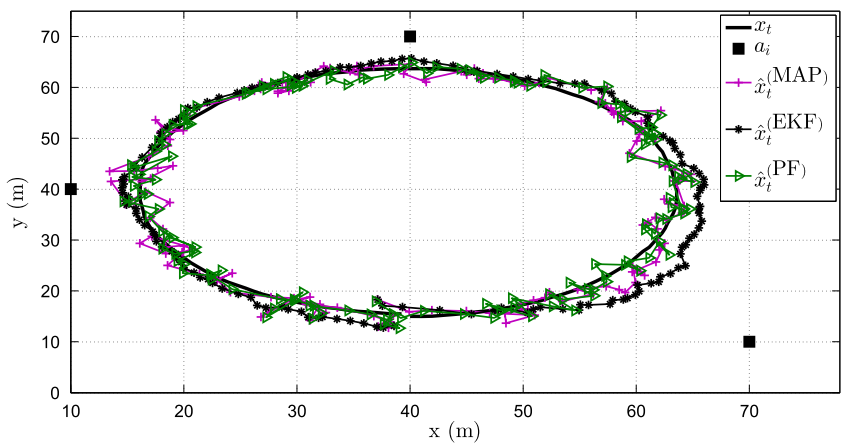

(b) Via MAP, EKF and PF.

Fig. 3. The true target trajectory in scenario 2, compared with the estimated trajectory.

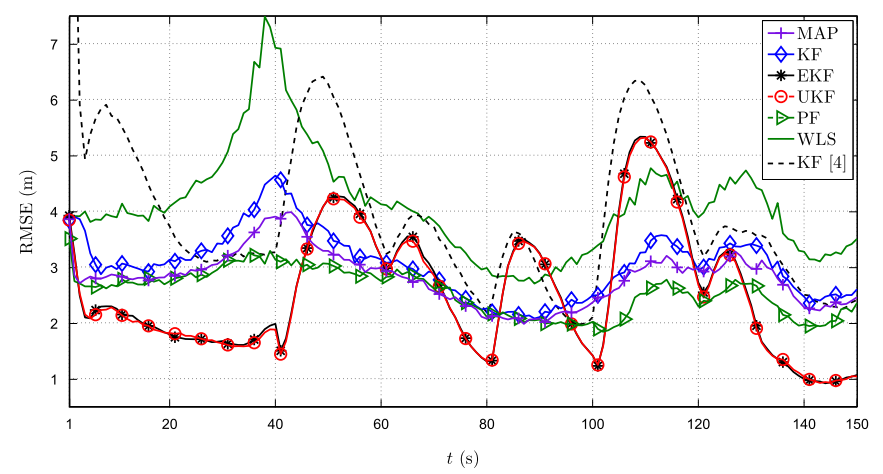

Fig. 4. RMSE versus $t$ (s) comparison, when $\sigma_{n_{i}}=9 \mathrm{~dB}, \sigma_{m_{i}}=4$ degrees, $\gamma=3$, $P_{0}=-10 \mathrm{dBm}, d_{0}=1 \mathrm{~m}, q=2.5 \times 10^{-3} \mathrm{~m}^{2} / \mathrm{s}^{3}, M_{c}=1000$.

general. Once again, modest performance deteriorations are observed in the proximity of any anchor.

Fig. 4 illustrates the RMSE versus $t$ comparison in the first considered scenario. The figure exhibits that the tracking algorithms, in general, perform better than the sequential localization one, as expected. Essentially, only at the critical points is where the localization algorithm possibly has advantage in comparison with the tracking ones. Moreover, Fig. 4 shows that the PF has the most stable performance, while the proposed KF outperforms the one in [4], suggesting that the new linearization technique offers considerable gain.

Fig. 5 illustrates the RMSE versus $t$ comparison in the second considered scenario. From this figure, it is absolutely clear that combining the prior knowledge with observations can significantly improve the estimation accuracy. This observation is in line with 


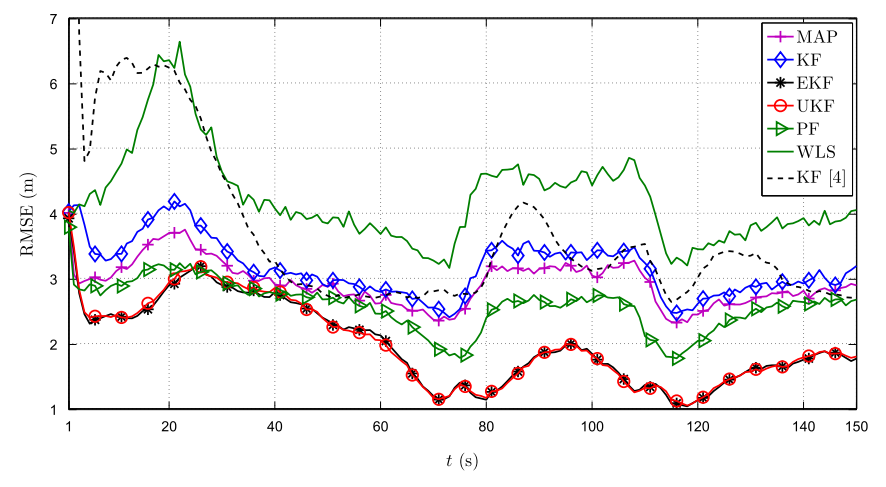

Fig. 5. RMSE versus $t$ (s) comparison, when $\sigma_{n_{i}}=9 \mathrm{~dB}, \sigma_{m_{i}}=4$ degrees, $\gamma=3$, $P_{0}=-10 \mathrm{dBm}, d_{0}=1 \mathrm{~m}, q=2.5 \times 10^{-3} \mathrm{~m}^{2} / \mathrm{s}^{3}, M_{c}=1000$.

Table 1

Average RMSE ( $\mathrm{m}$ ) of the considered algorithms.

\begin{tabular}{lll}
\hline Algorithm & Scenario 1 & Scenario 2 \\
\hline MAP & 2.86 & 2.96 \\
KF & 3.12 & 3.20 \\
EKF & 2.80 & 2.09 \\
UKF & 2.78 & 2.10 \\
PF, $q=2.5 \times 10^{-1} \mathrm{~m}^{2} / \mathrm{s}^{3}$ & 2.60 & 2.62 \\
PF, $q=2.5 \times 10^{-3} \mathrm{~m}^{2} / \mathrm{s}^{3}$ & 37.41 & 31.98 \\
KF $[4]$ & 4.22 & 4.30 \\
WLS & 4.31 & 4.14 \\
\hline
\end{tabular}

expectations, since the target trajectory is fairly smooth. Fig. 5 also exposes the proposed EKF and UKF algorithms as the best options for this scenario, and confirms the superiority of the proposed $\mathrm{KF}$ over the one in [4].

Finally, the average RMSE performance of the considered algorithms for both scenarios is presented in Table 1. In order to show the dependence of the PF on the tuning factor $q$, the results of $P F$ for two different values of $q$ are presented. Obviously, the PF is highly reliant on $q$, such that higher values of $q$ favor its performance. This is not completely surprising, since higher values of the tuning factor allow greater search space for particle deployment. From the table, we can also observe that the proposed KF outperforms the KF in [4] for about $1 \mathrm{~m}$. Furthermore, it can be seen that the proposed EKF and UKF have similar performance, and that, unlike the other algorithms, they significantly improve their performance in the second scenario, in comparison with the first one. This behavior is somewhat anticipated for EKF, which is a derivative-based estimation technique; thus, naturally favors mild target trajectories. In the case of UKF, although the mean and covariance are accurately captured with the discrete distribution with finite samples up to the 3rd order terms, its performance still depends on the higher order terms, and it is known to be scenariodependent ${ }^{6}$ in general $[22,23]$.

It is also worth mentioning that, in terms of computational complexity, the most expensive operations of an algorithm are matrix multiplication and inversion [26]. These operations are a part of practically all considered algorithms (except for the PF, but this algorithm has other drawbacks related with the number of

\footnotetext{
6 Note that the EKF algorithm might not be well approximated by the firstorder Taylor series expansion in the case of error propagation [22,23], and that the UKF algorithm depends on some heuristically chosen values (e.g., the parameter $\kappa$ in [20] which scales the sigma points of the unscented transformation towards or away from the mean of the prior distribution). Thus, the two algorithms might not converge always, as was the case in the considered scenarios. However, the results presented here are all for $M_{c}=1000$ cases in which they converged.
}

Table 2

Running time (s) of the considered algorithms for $M_{c}=1000$. Processor: Intel(R)Core(TM)i7-4710HQ, CPU@ 2.50 GHz.

\begin{tabular}{llllllll}
\hline Algorithm & MAP & KF & EKF & UKF & PF & WLS & KF [4] \\
\hline Scenario 1 & 36.0 & 31.3 & 37.0 & 54.1 & 467.6 & 28.8 & 31.2 \\
Scenario 2 & 36.1 & 31.4 & 37.1 & 55.2 & 468.9 & 28.8 & 31.9 \\
\hline
\end{tabular}

particles used), and it can be said that essentially all considered algorithms are of the same order of complexity. Nevertheless, the UKF and the PF use a number of sigma points and particles respectively, which affect their computational complexities and consequently their execution times, as it is shown in Table 2.

In Table 2, the running times (s) of the considered algorithms for both scenarios for $M_{c}=1000$ are presented. From it, one can see that the PF requires the longest running time of all algorithms, which is one order of magnitude longer than the running time of the remaining algorithms. This is not surprising, since the PF algorithm uses a large number of particles, which significantly increases its computational load. Similarly, the running time of the UKF is the second longest, which is a result of using sigma points in its execution. The running time of the remaining algorithms is practically the same. However it is worth pointing out that the EKF is a derivative-based algorithm, and finding the derivative can be very difficult or event not possible in some cases.

\section{Conclusions and future work}

In this work, the target tracking problem that utilizes hybrid (RSS and AoA) measurements was addressed. We have presented a novel linearization technique to approximate the highly nonlinear measurement model into a linear one. Extensive study of the problem based on Bayesian methodology has been carried out, which resulted in the proposal of two novel tracking algorithms, namely MAP and KF. Moreover, to the best of our knowledge, the application of the EKF and UKF algorithms for efficient solving of the considered RSS-AoA target tracking problem was presented here for the first time. Furthermore, the well-known $\mathrm{PF}$ algorithm was also presented. All of the presented algorithms take advantage of the prior knowledge, extracted from the state model, which is complemented with the observation information in order to enhance the estimation accuracy. The new algorithms were compared with the existing $\mathrm{KF}$ algorithm and the classical localization algorithm that neglects the prior knowledge in two different scenarios: where the target takes sharp maneuvers and a more smooth one. The simulation results have confirmed that employing the prior knowledge into an estimator can significantly reduce the estimation error. Moreover, the simulation results have confirmed that the proposed algorithms efficiently solve the target tracking problem, and that the proposed linearization technique used in the new KF offers considerably better estimation accuracy in comparison with the existing one in [4].

It should be pointed out that this work considered the tracking problem where the anchors were assumed static. A different approach in which the anchors are mobile and move in a certain direction based on pre-established rules that take into account the target estimate is left for future work. In this way, we hope to further improve the estimation accuracy of our algorithms and possibly reduce the number of anchors required for efficient problem solving.

\section{Acknowledgments}

This work was partially supported by Fundação para a Ciência e a Tecnologia under Project PEst-OE/EEI/UI0066/2014 (UNINOVA), 
Project UID/EEA/50008/2013 (Instituto de Telecomunicações), and Program Investigador FCT under Grant IF/00325/2015 and Grant SFRH/BD/91126/2012. M. Tuba is supported by the Ministry of Education, Science and Technological Development of Republic of Serbia, Grant No. III-44006.

\section{References}

[1] D. Dardari, P. Closas, P.M. Djuric, Indoor tracking: theory, methods, and technologies, IEEE Trans. Veh. Technol. 64 (4) (2016) 1263-1278.

[2] J.P. Beaudeau, M.F. Bugallo, P.M. Djuric, RSSI-based multi-target tracking by cooperative agents using fusion of cross-target information, IEEE Trans. Signal Process. 63 (19) (2015) 5033-5044.

[3] E. Masazade, R. Niu, P.K. Varshney, Dynamic bit allocation for object tracking in wireless sensor networks, IEEE Trans. Signal Process. 60 (10) (2012) 50485063.

[4] M.W. Khan, A.H. Kemp, N. Salman, L.S. Mihaylova, Tracking of wireless mobile nodes in the presence of unknown path-loss characteristics, in : Proc. Fusion, Washington DC, USA, Jul. 2015, pp. 104-111.

[5] M.W. Khan, N. Salman, A. Ali, A.M. Khan, A.H. Kemp, A Comparative study of target tracking with Kalman filter, extended Kalman filter and particle filter using received signal strength measurements, in: Proc. ICET, Peshawar, Pakistan, Dec. 2015, pp. 1-6.

[6] A. Lohbihler, Method and Apparatus for Position Sensing, Google Patents, US Patent App. 10/523,806, Jul. 2006. Available online: https://www.google.com/ patents/US20060166681.

[7] S. Tomic, M. Beko, R. Dinis, Distributed RSS-based localization in wireless sensor networks based on second-order cone programming, Sensors 14 (10) (2014) 18410-18432.

[8] S. Tomic, M. Beko, R. Dinis, RSS-based localization in wireless sensor networks using convex relaxation: Noncooperative and cooperative schemes, IEEE Trans. Veh. Technol. 64 (5) (2015) 2037-2050.

[9] Y. Wanga, J. Huangb, L. Yangb, Y. Xue, TOA-based joint synchronization and source localization with random errors in sensor positions and sensor clock biases, Ad Hoc Networks 29 (2015) 99-111.

[10] J. Yia, D. Mirzab, R. Kastnerb, C. Schurgersc, P. Robertsd, J. Jaffe, ToA-TS: Time of arrival based joint time synchronization and tracking for mobile underwater systems, Ad Hoc Networks 29 (2015) 99-111.

[11] S. Tomic, M. Beko, R. Dinis, 3-D target localization in wireless sensor network using RSS and AoA measurement, IEEE Trans. Veh. Technol. 66 (4) (2017) 3197-3210.

[12] S. Tomic, M. Beko, R. Dinis, Distributed RSS-AoA based localization with unknown transmit powers, IEEE Wirel. Commun. Lett. 5 (4) (2016) 392-395.

[13] A. Stanoev, S. Filiposka, V. In, L.J. Kocarev, Cooperative method for wireless sensor network localization, Ad Hoc Networks 40 (2016) 61-72.

[14] S. Tomic, M. Beko, R. Dinis, P. Montezuma, Distributed algorithm for target localization in wireless sensor networks using RSS and AoA Measurements Pervasive Mob. Comput. 37 (2017) 63-77.

[15] S. Tomic, M. Beko, R. Dinis, P. Montezuma, A closed-form solution for RSS/AoA Target Localization by Spherical Coordinates Conversion, IEEE Wirel. Commun. Lett. 5 (6) (2016) 680-683.

[16] S.M. Kay, Fundamentals of Statistical Signal Processing: Estimation Theory, Prentice-Hall, Upper Saddle River, NJ, USA, 1993.

[17] G. Wang, Y. Li, M. Jin, On MAP-based target tracking using range-only measurements, in: Proc. CHINACOM, Guilin, China, Aug. 2013, pp. 1-6.

[18] T.S. Rappaport, Wireless Communications: Principles and Practice, PrenticeHall, Upper Saddle River, NJ, USA, 1996.

[19] K. Yu, 3-D localization error analysis in wireless networks, IEEE Trans. Wirel. Commun. 6 (10) (2007) 3473-3481.

[20] S.J. Julier, J.K. Uhlmann, Unscented filtering and nonlinear estimation, Proc. IEEE 92 (3) (2004) 401-422.

[21] E.A. Wan, R. Van Der Merwe, The unscented Kalman filter for nonlinear estimation, in: Proc. IEEE AS-SPCC, Lake Louise, Alberta, Canada, Oct. 2000, pp $1-6$.

[22] Z. Zhao, H. Chen, G. Chen, C. Kwan, X.R. Li, Comparison of several ballistic target tracking filters, in: Proc. ACC, Minneapolis, MN, USA, Jun. 2006, pp. 2197-2202.

[23] A. Farina, B. Ristic, D. Benvenuti, Tracking a ballistic target: comparison of several nonlinear filters, IEEE Trans. Aerosp. Electron. Syst. 38 (3) (2002) 854 867.

[24] E.N. Chatzi, A.W. Smyth, The unscented Kalman Filter and particle filter methods for nonlinear structural system identification with non-collocated heterogeneous sensing, Struct. Control Health Monit. 16 (1) (2009) 99-123.

[25] Z. Chen, Bayesian filtering: from Kalman filters to particle filters, and beyond, Statistics 182 (1) (2003) 1-69.

[26] L.M. Paz, J.D. Tardós, J. Neira, Divide and conquer: EKF SLAM in $\mathcal{O}(n)$, IEEE Trans. Robot. 24 (5) (2008) 1107-1120.

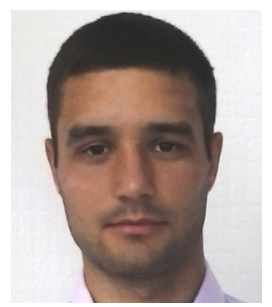

Slavisa Tomic received the M.S. degree in traffic engineering according to the postal traffic and telecommunications study program from University of Novi Sad, Serbia in 2010, and the Ph.D. degree in electrical and computer engineering from University Nova of Lisbon, Portugal in 2017. His research interests include target localization in wireless sensor networks, and non-linear and convex optimization.

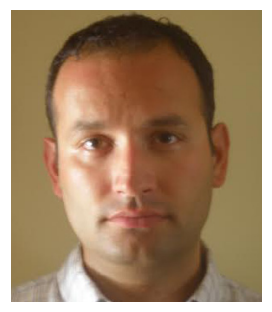

Marko Beko was born in Belgrade, Serbia, on November 11,1977 . He received the Dipl. Eng. degree from the University of Belgrade, Belgrade, Serbia, in 2001 and the Ph.D. degree in electrical and computer engineering from Instituto Superior Tecnico, Lisbon, Portugal, in 2008. Currently, he is an Associate Professor at the Universidade Lusfona de Humanidades e Tecnologias, Portugal. He is also a Researcher at the UNINOVA, Campus da FCT/UNL, Monte de Caparica, Portugal. His current research interests are in the area of signal processing for wireless communications and nonsmooth and convex optimization. He serves as an Associate Editor for the IEEE Access Journal and Elsevier Journal on Physical Communication. He was awarded a Starting Grant under the Investigador FCT programme of the Portuguese Science and Technology Foundation in 2016. He is the winner of the 2008 IBM Portugal Scientific Award.

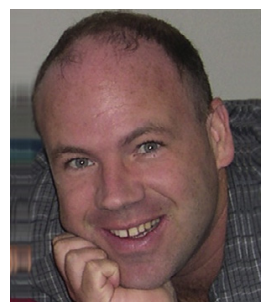

IEEE Member (2000) Rui Dinis (M'00, SM'14) received the Ph.D. degree from Instituto Superior Técnico (IST), Technical University of Lisbon, Portugal, in 2001 and the Habilitation in Telecommunications from Faculdade de Ciências e Tecnologia (FCT), Universidade Nova de Lisboa (UNL), in 2010. From 2001 to 2008 he was a Professor at IST. Currently he is an associated professor at FCTUNL. During 2003 he was an invited professor at Carleton University, Ottawa, Canada.

He was a researcher at CAPS (Centro de Análise e Processamento de Sinal), IST, from 1992 to 2005 and a researcher at ISR (Instituto de Sistemas e Robótica) from 2005 to 2008. Since 2009 he is a researcher at IT (Instituto de Telecomunicações).

Rui Dinis is editor at IEEE Transactions on Communications (Transmission Systems--Frequency-Domain Processing and Equalization) since 2012 and editor at IEEE Transactions on Vehicular Technology since 2015. He was also a guest editor for Elsevier Physical Communication (Special Issue on Broadband Single-Carrier Transmission Techniques). Rui Dinis was TPC chair of the IEEE ICT'2014. He has been actively involved in several international research projects in the broadband wireless communications area.

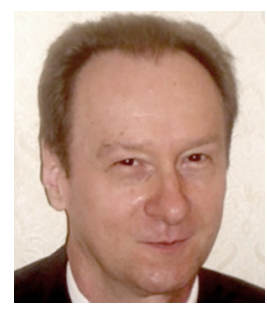

Milan Tuba is the Dean of Graduate School of Computer Science and Provost for mathematical and technical sciences at John Naisbitt University of Belgrade. He received B.S. in Mathematics, M.S. in Mathematics, M.S. in Computer Science, M. Ph. in Computer Science, Ph.D. in Computer Science from University of Belgrade and New York University. From 1983 to 1994 he was in the U.S.A. at Vanderbilt University in Nashville and Courant Institute of Mathematical Sciences, New York University and later as Assistant Professor of Electrical Engineering at Cooper Union School of Engineering, New York. From 1994 he was Professor of Computer Science and Director of Computer Center at University of Belgrade and from 2004 also a Professor of Computer Science and Dean of the College of Computer Science, Megatrend University Belgrade. His research interest includes heuristic optimizations applied to computer networks, image processing and combinatorial problems. Prof. Tuba is the author or coauthor of more than 150 scientific papers and coeditor or member of the editorial board or scientific committee of number of scientific journals and conferences. Member of the ACM, IEEE, AMS, SIAM, IFNA. 


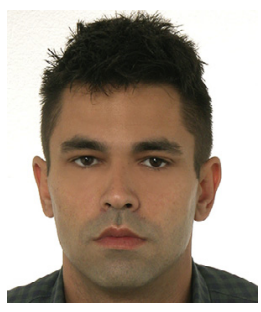

Nebojsa Bacanin was born in 1983 in Belgrade. In 2015 he completed Ph.D. studies in Computer Science at the Faculty of Mathematics, University of Belgrade. Currently he is an assistant professor and vice dean at the Faculty of Computer Science at John Naisbitt University.

He has held classes in several courses at the undergraduate and graduate studies, from operating systems and computer networks to databases and programming. He has been engaged in the project III-44006 from the program of integrated inter-disciplinary researches of the Ministry of Education, Science and Technological Devel- opment of the Republic of Serbia. His scientific contribution extends to the optimization and metaheuristic methods based on the intelligence of swarms (swarm intelligence), both for continuous, and for discrete problems. He has published numerous scientific papers in leading international journals indexed in the $\mathrm{SCl}$ list (Thomson Reuters ISI), Scopus and/or MathSciNet, as well as, at international conferences indexed in Web of Science, Scopus, and/or IEEE Xplore. He is a regular reviewer of many of the leading top international journals and he participated in organizing committees of the worlds leading conferences. 